06

\title{
Взрывной переход в аморфном микропроводе
}

\author{
() И.Ю. Борисенко, В.А. Тулин \\ Институт проблем технологии микроэлектроники и особочистых материалов РАН, \\ Черноголовка, Московская обл., Россия \\ e-mail: eagle@iptm.ru
}

(Поступило в Редакцию 9 марта 2017 г.)

Аморфный микропровод в стеклянной оболочке обладает малой тепловой инерцией и может быть быстро нагрет до температуры кристаллизации. Мы наблюдали процесс перехода микропровода из аморфного в микрокристаллическое состояние при нагреве его импульсным током разной амплитуды и длительности. Процесс кристаллизации аморфного состояния происходил двумя путями в зависимости от параметров импульса: „медленным“ и взрывным. Во втором случае наблюдалось излучение электромагнитных волн (вспышка света) и всплеск сопротивления. Скорость распространения фронта кристаллизации в наших экспериментах составляла около $1 \mathrm{~m} / \mathrm{s}$.

DOI: 10.21883/JTF.2017.11.45128.2236

Изучению явления взрывной кристаллизации (ВК) аморфных материалов уделено значительное внимание исследователей в области химии, физики, материаловедения и других наук. Аморфные вещества представляют собой неравновесные „замороженные“ метастабильные состояния. При повышении температуры энергетический барьер, отделяющий это состояние от равновесного микрокристаллического, может преодолеваться, и появляется возможность перехода в равновесное нанокристаллическое состояние. Отличительной чертой этого перехода является возможность существования наряду с медленными и быстрых процессов, связанных с распадом на фазы и с движением фронта кристаллизации за счет его значительного саморазогрева [1].

Скорость роста кристаллической фазы в первоначальном режиме, связанным с преодолением потенциального барьера, описывается аррениусовской зависимостью

$$
U=U_{0} \exp (-E / k T),
$$

где $U_{0}-$ константа, $E-$ энергия активации, $k-$ постоянная Больцмана и $T$ - абсолютная температура. Согласно этому выражению, значение температуры кристаллизации не является строго определенным. Процесс роста идет с достаточно заметной скоростью в некотором диапазоне температур. Поскольку реакция превращения аморфного материала в микрокристаллический является экзотермической, она характеризуется некоторым удельным тепловыделением $Q$. Масштаб этого тепловыделения играет большую роль в определении характера перехода. Если тепловыделение в процессе перехода меньше теплоотдачи образца в окружающую среду, то переход происходит „однородно“ в объеме образца с постепенным понижением степени неравновесности аморфного материала (процесс близок к изотермическому). В противном случае (взрывная кристаллизация) выделение скрытой теплоты фазового превращения приводит к локальному нагреву образца, к ускорению процесса перехода, к распаду на фазы и к возникновению фазовых границ. Тепловыделение сосредоточивается на границе раздела фаз, фронт кристаллизации саморазогревается и устремляется в сторону аморфного материала, переводя его в микрокристаллическое состояние. При этом скорость фронта кристаллизации в самоподдерживающем режиме может достигать $32 \mathrm{~m} / \mathrm{s}$ [2,3].

ВК наблюдалась в аморфных пленочных структурах: полупроводниках [4-6], металлах [7,8], диэлектриках [9]. Большую роль при этом играла подложка, в которую осуществлялся основной отвод тепла, выделяющегося при саморазогреве. Образцы в виде аморфных пленок удобны для исследований, так как они позволяют наблюдать за процессом превращения, используя различные микроскопы [5,10,11]. Пространственные масштабы там вполне макроскопические (миллиметры, сантиметры). Мы же исследовали процесс фазового перехода в аморфном микропроводе. Аморфный микропровод, полученный методом Улитовского-Тейлора, является достаточно важным высокотехнологичным материалом с точки зрения приложений. Особенно это касается его магнитных и микроволновых свойств. В послевоенные годы технология, развитая Улитовским, позволила резко улучшить качество гальванометров, в то время основных приборов изучения электрических и магнитных свойств материалов.

В настоящей работе мы исследовали микропровод состава $\mathrm{Fe}_{15} \mathrm{Co}_{60} \mathrm{~B}_{10} \mathrm{Si}_{15}$. Куски этого провода остались у нас после выполнения работы [12] с сотрудниками Института стали и сплавов. Диаметр металлического проводника составлял $16 \mu \mathrm{m}$, диаметр стеклянного покрытия (оболочки) $26 \mu \mathrm{m}$. Для разогрева микропровода мы использовали импульсный источник тока (импульс прямоугольной формы длительностью от 20 до $500 \mathrm{~ms}$ ). Отрезок микропровода длиной 6-10 mm монтировался в капли индий-галлиевого сплава (жидкого при комнатной температуре), служащие контактами на электрической разводке, выполненной на фольгированном текстолите. 


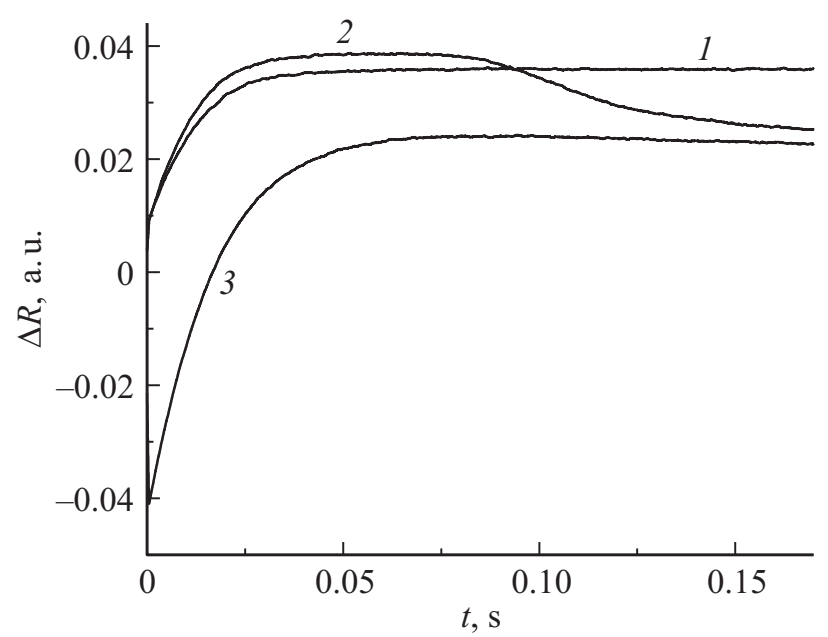

Рис. 1. Примеры регистрации зависимости изменения сопротивления от времени, прошедшего от начала токового импульса.

При измерениях на осциллографе контролировались: изменение падения напряжения на образце (использовалась мостовая схема), ток через образец и выходное напряжение источника (генератора импульсов).

В качестве метода характеризации состояния микропровода мы использовали наблюдение ферромагнитного резонанса (ФМР). С этой целью мы провели наблюдение ФМР в микропроводе, находящемся в аморфном или микрокристаллическом состоянии. В аморфном состоянии ФМР легко наблюдается в единичных отрезках микропровода длиной 2-3 mm (при правильном их расположении в резонаторе) [12]. В микрокристаллическом состоянии ФМР практически не наблюдался в подобных отрезках микропровода при измерении на нашем ЭПРспектрометре.

На рис. 1 изображены зависимости величины изменения сопротивления от времени, отсчитываемого от начала токового импульса. Кривые 1 и 2 отвечают нарастанию амплитуды токового импульса. Кривые 2 и 3 зарегистрированы при том же самом выходном импульсе генератора. После прохождения процесса, соответствующего кривой 1 , образец остается в аморфном состоянии (ФМР после такой обработки наблюдается). После прохождения кривой 2 образец переходит в новое состояние (отсутствие ФМР). Кривая 3 соответствует процессу воздействия токового импульса на образец в новом состоянии. После включения импульса микропровод начинает разогреваться до температуры, определяемой амплитудой тока в импульсе. Тепловое время образца составляло около $20 \mathrm{~ms}$, т. е. за время $50 \mathrm{~ms}$ образец практически достигал равновесной температуры, соответствующей амплитуде тока в импульсе. Это хорошо видно на кривых, где не происходит радикальных изменений (кривые 1 и 3). Кривая 2 заметно отличается от вышеупомянутых кривых. На ней наблюдается излом, после которого сопротивление образца начинает падать во времени. Процесс излома на зависимости сопротивления от температуры происходит за время порядка $40 \mathrm{~ms}$, после чего происходит установление равновесия в новой системе (экспоненциальное уменьшение температуры в соответствии с новым значением сопротивления). Многочисленные повторения подобных опытов показали, что, как только при воздействии токового импульса мы наблюдаем временную зависимость типа 2 (излом), образец меняет свои свойства. При этих измерениях было замечено, что в процессе нагрева в момент вышеупомянутого излома микропровод, если он наблюдается во временных пределах $100 \mathrm{~ms}$ от начала импульса, кратковременно (в течение времени значительно короче длительности токового импульса) излучает свет. Конечно, длительность вспышки мы определили уже после проведения соответствующих измерений.

Мы добавили четвертый канал измерений - измерение интенсивности света. С этой целью мы разместили рядом с микропроводом, на расстоянии 3-4 mm, фотодиод OPT-211 фирмы BURR-BROWN, поместили измерительную часть в закрытую коробку и во время действия токового импульса фиксировали излучение из микропровода. На рис. 2 представлен пример записи сигналов световой эмиссии (кривая 1) и изменения сопротивления образца (кривая 2) во время действия токового импульса. От начала токового импульса микропровод нагревается за счет джоулева тепла. При этом его сопротивление возрастает. В качестве опорной кривой на рис. 2 нанесена зависимость изменения падения напряжения на том же самом образце (его сопротивления) после нагрева до более высокой температуры, когда процессы структурной перестройки завершились (кривая 3). Масштаб кривой 3 изменен до совмещения начальных участков кривых 2 и 3 . При нагреве до некоторой температуры изменение сопротивления образца отстает от опорной кривой и даже начинает умень-

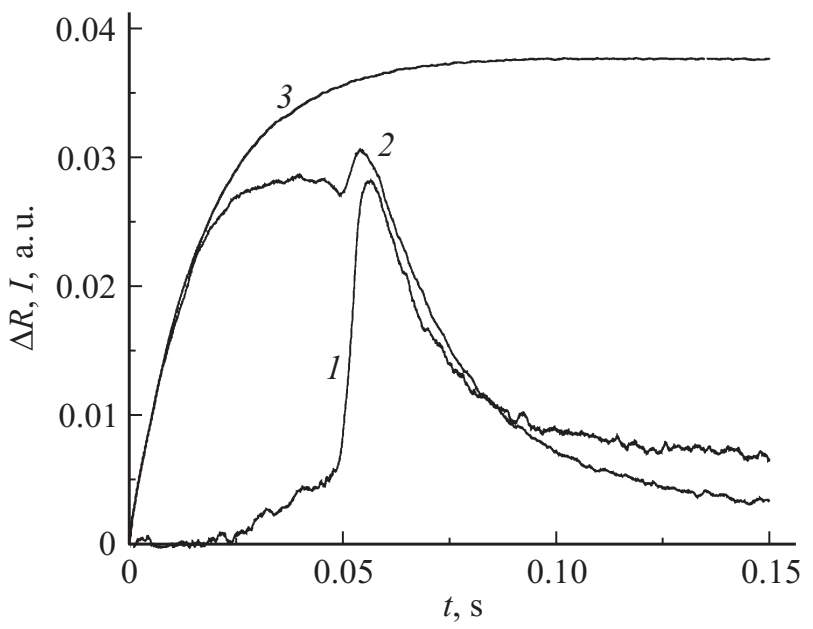

Рис. 2. Изменение яркости свечения (1) и сопротивления (2) от времени, прошедшего от начала токового импульса. Изменение сопротивления образца (3), претерпевшего структурный переход ранее. 
шаться. В образце начинают происходить структурные изменения, приводящие к падению его сопротивления. Затем разгорается вспышка света, сопротивление в этот момент также имеет всплеск и затем спадает во времени. Время разгорания вспышки составляет около $5 \mathrm{~ms}$. На самом деле, более слабое фоновое свечение начинается немного раньше по времени и остается после окончания вспышки. Это, по-видимому, обычное тепловое излучение, соответствующее температуре микропровода при подходе к взрывному переходу материала в микрокристаллическое состояние $\left(\sim 400^{\circ} \mathrm{C}\right)$, которое чувствует фотодиод.

Здесь стоит обратить внимание на трудности, с которыми сталкиваешься при выполнении этих измерений. Хотя процесс нагрева образца импульсным током достаточно гибок, можно менять ток достаточно точно и плавно, переход из аморфного состояния в микрокристаллическое является необратимым, т.е. на каждом образце его можно наблюдать только один раз. Вследствие имеющего место небольшого разброса параметров микропровода (прежде всего площади поперечного сечения) трудно точно установить желаемое значение тока, необходимое для контролируемого воздействия. С одной стороны, приходится часто перемонтировать образцы, что само по себе является достаточно кропотливой работой, с другой - в начальный момент мы не знаем значения критического тока, при котором образец нагреется до или выше температуры взрывного перехода в кристаллическое состояние. Величина этого тока для отрезков микропровода из разных участков менялась от 50 до $60 \mathrm{~mA}$ (плотность критического тока около $2 \cdot 10^{4} \mathrm{~A} / \mathrm{cm}^{2}$ ). После того, как мы превысили температуру перехода, мы получили нужную информацию, интерес к образцу пропадает, и нужно монтировать новый. Если же нам не удалось перевести образец в новое состояние первым импульсом, то, как видно из рис. 2, в нем происходят некоторые структурные изменения, и аморфность образца (количество аморфной фазы) уменьшается.

На рис. 3 представлен набор кривых, регистрирующих сигналы всплесков свечения, наблюдаемые для пяти образцов микропровода, в которых переход наблюдался при первом включении импульса тока. Здесь видно, что интенсивность свечения уменьшается пропорционально промежутку времени от начала импульса, хотя временные характеристики излучения остаются подобными (как возгорание, так и затухание). Свечение разгорается до максимальной амплитуды за $5 \mathrm{~ms}$, а спадает за время тепловой инерции образца. Изменение интенсивности фонового свечения для разных опытов (амплитуда сигнала в правой части рисунка) говорит о различии конечной температуры в каждом измерении. Если излом сопротивления происходит во временном интервале по прошествии $100 \mathrm{~ms}$ после начала импульса, то заметного всплеска свечения не наблюдается (кривая 1).

Аморфное состояние материала является метастабильным. Его существование при обычных температурах

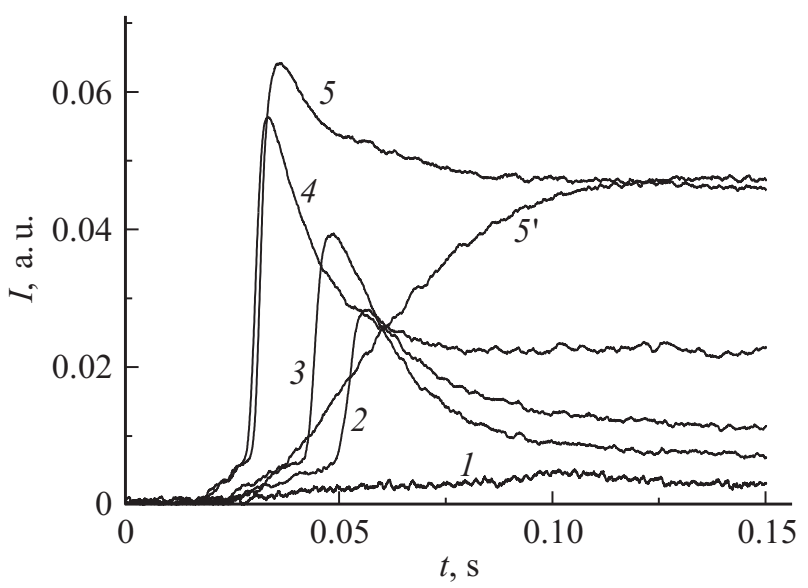

Рис. 3. Примеры изменения яркости свечения во время воздействия токового импульса закритической амплитуды: 1 докритическая амплитуда (пояснение в тексте); 2-5 - закритическая амплитуда (возрастает с ростом номера ); $5^{\prime}-$ свечение ранее перешедшего образца при амплитуде соответствует 5 .

определяется наличием достаточно большого энергетического барьера, отделяющего аморфное состояние от микрокристаллического. При повышении температуры этот барьер начинает преодолеваться активационным образом, и материал локально претерпевает изменения, приближаясь к микрокристаллическому состоянию. Переход является экзотермическим и сопровождается тепловыделением, что может стимулировать распространение перехода на соседнюю часть образца. При определенном соотношении между этим тепловыделением и теплоотдачей в окружающую среду начинается самораспростронение фронта микрокристаллического состояния, и материал спонтанно переходит в это состояние. В литературе это получило название взрывного перехода. При этом образец нагревается, и может возникнуть дополнительное тепловое излучение сверх фонового, связанного с высокой стартовой температурой образца. Несколько ниже этого критического условия переход происходит почти изотермически без характерных для взрывного перехода особенностей. Атомы передвигаются в свои равновесные позиции независимо (не лавинообразно), и материал кристаллизуется с „меньшей“ скоростью. Переход от одного режима к другому может происходить при увеличении объемной скорости кристаллизации (1), когда теплоотвод не может поглотить выделяющееся тепло. Как можно видеть на рис. 3, фоновое излучение достигает определенного уровня перед началом вспышки. Характеристика фонового излучения в нашем случае - это отклик фотодиода на тепловое излучение нагретого микропровода, т.е. на его температуру. Процесс взрывного перехода начинается в нашем случае при достижении микропроводом определенной температуры. 
Исходя из этих рассуждений, можно понять большинство результатов, полученных в настоящей работе. Если мы при первом измерении установили ток в импульсе меньше критического, то в образце происходит медленный процесс перехода в кристаллическое состояние. Так как температура образца при этом всетаки близка к критической, процесс идет достаточно быстро, и по окончанию импульса может остаться малое количество аморфной фазы. Последующий импульс приведет к ослабленному взрывному переходу или к его замедлению, вплоть до полного отсутствия. Поэтому интересны данные, когда взрывной переход происходит при первом включении импульса тока. Хотя и в этом случае часть образца успевает перейти в кристаллическое состояние за время, прошедшее от начала импульса до момента начала взрывного перехода. Это хорошо видно на рис. 2, где сопротивление начинает активно уменьшаться, несмотря на рост температуры. С этим связана зависимость амплитуды световой вспышки от времени, прошедшего с начала импульса, и ненаблюдаемость вспышки по истечению $100 \mathrm{~ms}$ от начала импульса.

Два наблюдаемых эффекта, по нашему мнению, могли представлять интерес для выяснения строения фронта кристаллизации - это всплеск свечения образца и одновременное с ним возрастание сопротивления. Из наблюдений взрывной кристаллизации в аморфных пленках известно, что фронт кристаллизации выглядит как линия, т. е. ширина его много меньше длины (в развитом состоянии). При этом яркость его свечения заметно превосходит фоновое свечение остальной пленки (как до его прохождения, так и после). Случай аморфного микропровода, полученного методом Улитовского-Тейлора, представляет определенный интерес тем, что он является квазиодномерным объектом (в нашем случае длина 5-10 mm, диаметр $0.016 \mathrm{~mm})$. Фронт кристаллизации в основной стадии развития движется в нем вдоль оси провода, плоскость фронта перпендикулярна оси. Это подтверждают выполненные измерения. Мы изучали больше десятка образцов, и большинство из них демонстрировали однотипное поведение (как на рис. 2). Только в единичных случаях вспышка была двойная или размазанная, видимо, в этих образцах наблюдались два или больше центров зарождения кристаллической фазы. Задача о теплопередачи в случае микропровода также упрощена, она сводится к обмену тепла между стеклянной оболочкой и окружающим газом.

Сигнал вспышки (точнее выходное напряжение фотодиода) сначала нарастает со временем порядка $5 \mathrm{~ms}$ практически линейно, а затем спадает со временем тепловой инерции. По нашим представлениям это соответствует накоплению тепла в образце при движении фронта кристаллизации с последующим остыванием после окончания процесса. Между процессом взрывного перехода (выделением скрытого тепла) и фиксацией сигнала фотодиода много непростых преобразований. Но если бы

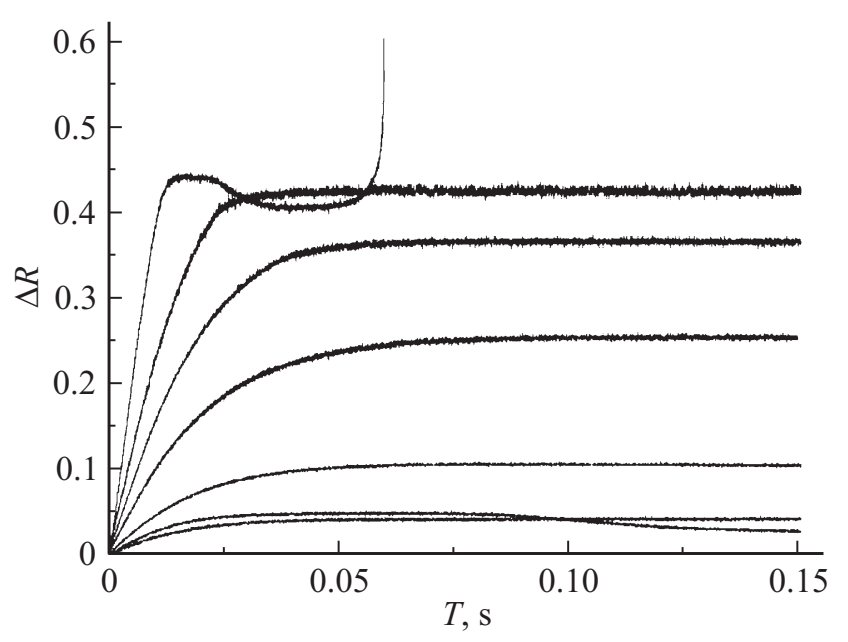

Рис. 4. Изменение сопротивления образца под воздействием токовых импульсов разной амплитуды.

за сигнал с фотодиода был ответствен фронт кристаллизации, выходное напряжение было бы близко к постоянному значению. Видно, что характер всех наблюдаемых переходов для разных образцов на стадиях роста и падения сигнала подобны (рис. 2). Это соответствует для наших квазиодномерных образцов простейшей картине процесса. Переход зарождается где-то в средней точке открытого провода и распространяется оттуда в обе стороны до мест вхождения провода в индий-галлиевый припой. Что происходит внутри припоя, представить довольно трудно, тепловые условия там отличаются. В связи с этим можно вернуться к данным рис. 1, где показан плавный процесс перехода, без вспышки. Видно, что в процессе перехода сопротивления образца уменьшаются ниже уровня предыдущей записи, когда переход не происходил. Казалось бы, джоулево тепло стало заметно меньше, чем требуется для продолжения развития перехода, однако переход продолжает идти в том же темпе. На этом основании можно предположить, что фронт перехода может распространяться в микропроводе и внутри припоя. Это определяет трудности с оценкой изменения электросопротивления образцов. В целом такой характер перехода подразумевает происхождение вспышки (сигнала, фиксируемого фотодиодом) из-за нагрева образца теплом, выделяющимся при прохождении фронта кристаллизации, а не излучением самого фронта.

Теперь перейдем к обсуждению результатов по измерению электросопротивления. Здесь будет уместно привести расширенные данные по этим измерениям. Микропровод, полученный методом Улитовского-Тейлора, представляет определенный демонстрационный интерес для наблюдения температурного изменения сопротивления. Дело в том, что он покрыт стеклянной оболочкой, которая разрушается при температуре выше точки плавления материала провода. На рис. 1 в процессе записи кривых 2 и 3 не проводилась компенсация 
сопротивления образца при комнатной температуре. Там видно, что сопротивление образца в микрокристаллическом состоянии значительно меньше сопротивления в аморфном. В то время как $d R / d T$ (где $R-$ сопротивление микропровода и $T$ - температура) в аморфном состоянии меньше, чем в кристаллическом. Разница сопротивлений при комнатной температуре составляет величину порядка $20 \%$ от полного сопротивления образца. Если, например, сопротивление образца в аморфном состоянии $40 \Omega$, то в микрокристаллическом оно составляет величину $32-34 \Omega$. Это характерные величины. Используя эти оценки и то обстоятельство, что мы не производили компенсацию нашей мостовой схемы измерений для приведенных зависимостей на рис. 1, можно представить все характерные значения изменений электросопротивления. На рис. 4 представлены результаты измерения сопротивления (в относительных единицах) при пропускании импульса тока различной амплитуды. Первые три кривые соответствуют данным на рис. 1, но для другого образца. Последующие кривые соответствуют увеличению амплитуды тока. На предпоследней кривой материал микропровода находится в состоянии плавления, максимальное сопротивление (температура) не меняется. На последней кривой материал расплавился (область постоянного сопротивления), в жидком состоянии сопротивление образца с ростом температуры уменьшается [13], затем разрушается стеклянная оболочка, жидкий материал провода вытекает, нарушая целостность провода, и сопротивление резко увеличивается. В разрушении целостности образца можно было убедиться после окончания эксперимента. Если теперь воспользоваться измерением сопротивления как термометром, считая, что сопротивление сильно неупорядоченного сплава меняется линейно с температурой [13], и грубо оценочно посчитать, что переход аморфное-микрокристаллическое состояние происходит при $400^{\circ} \mathrm{C}$, а плавление материала провода при $1000^{\circ} \mathrm{C}$, то из амплитуды всплеска сопротивления при переходе можно оценить средний нагрев образца (порядка $100^{\circ} \mathrm{C}$ ).

То есть наблюдение эффектов возникновения вспышки света и всплеска сопротивления можно объяснить простым нагревом, что не дает нам информации о структуре фронта взрывной кристаллизации. Есть еще надежда на изучение спектрального состава вспышки света. Дело в том, что в первых измерениях мы наблюдали вспышку света невооруженным глазом. Так вот, вспышка выглядела ярко белой, а не красной, как это должно быть при соответствующих температурах. К сожалению, мы не готовы к этим измерениям, нет необходимой оснастки и приборов.

Мы наблюдали взрывной переход из аморфного в микрокристаллическое состояние в микропроводе состава $\mathrm{Fe}_{15} \mathrm{Co}_{60} \mathrm{~B}_{10} \mathrm{Si}_{15}$, полученном методом Улитовского-Тейлора, при пропускании импульса тока. Взрывной характер перехода возникает при достижении образцом определенной температуры и сопровождается вспышкой света и кратковременным возрастанием электрического сопротивления. Наблюдаемые черты перехода: поведение среднего сопротивления образца и излучения, фиксируемого фотодиодом, могут быть объяснены тепловыми эффектами, связанными с экзотермическим характером процесса и скоростью джоулева разогрева образца. Скорость распространения фронта в наших экспериментах составляет величину около $1 \mathrm{~m} / \mathrm{s}$ (длина $5 \mathrm{~mm}$, время - $5 \mathrm{~ms}$ ). Несмотря на некоторую неопределенность в параметрах микропровода, можно достаточно плавно переходить от изотермического режима к взрывному, что дает дополнительные возможности в изучении самого процесса.

В заключение авторы выражают благодарность Р.В. Холину за большую помощь при проведении экспериментов и А.М. Орлову за разработку и изготовление измерительного блока.

\section{Список литературы}

[1] Шкловский В.А. // ЖЭТФ. 1982. Т.82. стр. 536.

[2] Палатник Л.С., Фукс М.Я., Косевич В.М. Механизм образования и субструктура конденсированных пленок. М.: Наука, 1972. 320 с.

[3] Ohdaira K., Matsumura H. // Thin Sol. Film. 2012. Vol. 524. P. $161-165$.

[4] Александров Л.Н. Кинетика кристаллизации и перекристаллизации полупроводниковых пленок. Новосибирск: Наука, 1985. 224 с.

[5] Шкловский В.А., Кузьменко В.М. // УФН. 1989. Т. 157. Вып. 2. C. 311-338.

[6] Nikolova L., Stern M.J., MacLeod J.M., Reed B.W., Ibrahim H., Campbell G.H., Rosei F., LaGrange T., Siwick B.J. // J. Appl. Phys. 2014. Vol. 116. P. 093512.

[7] Bostanjoglo O., Liedtke R. // Phys. Stat. Sol. Ser. A. 1980. Vol. 60. P. 451-455.

[8] Kuz'menko V.M., Chernyaeva T.P. // Low Temp. Phys. 2005. Vol. 31. N 2. P. 111-115.

[9] Скрипов В.П., Коверда В.П. Спонтанная кристаллизация переохлажденных жидкостей. М.: Наука, 1984. 232 с.

[10] Nikolova L., LaGrange T., Reed B.W., Stern M.J., Browning N.D., Campbell G.H., Kieffer J.C., Siwick B.J., Rosei F. // Appl. Phys. Lett. 2010. Vol. 97. N 20. P. 203102.

[11] Nikolova L., LaGrange T., Stern M.J., MacLeod J.M., Reed B.W., Ibrahim H., Campbell G.H., Rosei F., Siwick B.J. // Phys. Rev. B. 2013. Vol. 87. P. 064105.

[12] Tulin V.A., Astahov M.V., Rodin A.O. // J. Magn. Magn. Mater. 2003. Vol. 258, N S1. P. 201-203.

[13] Гантмахер В.Ф. Электроны в неупорядоченных средах. М.: Физматлит, 2003. 174 c. 